\title{
Effects of Habitat Modifications on Behavioral Indicators of Welfare for Madagascar Giant Hognose Snakes (Leioheterodon madagascariensis)
}

\author{
Marisa S. Spain ${ }^{* 1,2}$, Grace Fuller ${ }^{1}$, and Stephanie M. Allard ${ }^{1}$ \\ ${ }^{1}$ Detroit Zoological Society, Royal Oak, Michigan \\ ${ }^{2}$ Currently at Jacksonville Zoo and Gardens, Jacksonville, Florida \\ *Corresponding author (Email: spainmarisa@ outlook.com)
}

Citation - Spain, M. S., Fuller, G., \& Allard, S. M. (2020). Effects of habitat modifications on behavioral indicators of welfare for Madagascar giant hognose snakes (Leioheterodon madagascariensis). Animal Behavior and Cognition, 7(1), 70-81. doi: https://doi.org/10.26451/abc.07.01.06.2020

\begin{abstract}
Although historically understudied, the empirical evaluation of captive reptile welfare is becoming more common, and zoos continue making modifications to their reptile facilities with the goal of improving welfare. In this study, we evaluated the impacts of habitat modifications on the behavior of five Madagascar giant hognose snakes (Leioheterodon madagascariensis) housed in the Holden Reptile Conservation Center at the Detroit Zoo. The snakes' enclosures were modified from smaller stainless steel boxes with newspaper substrate and plastic hide boxes to larger enclosures with open glass fronts and naturalistic elements. Along with nearly doubling enclosure size, the new enclosures featured a deep sand/mulch mixture for substrate instead of newspaper, as well as corkbark furnishings in addition to the plastic hide boxes. We hypothesized that the modifications made to the enclosures would provide opportunities for the expression of species-typical behaviors, such as exploration, digging (an important foraging behavior for hognose species), and locomotion, as well as increased behavioral diversity and overall activity levels. Each individual was observed for a total of $7.7 \mathrm{hrs}$ with approximately $3.8 \mathrm{hrs}$ in both the baseline and modified conditions. Data were collected using both scan and all-occurrence sampling methods in 10min focal observation sessions, twice daily, three times a week. Generalized linear mixed models showed that the modified habitats increased behavioral diversity and environmental exploration compared to baseline data. One snake showed substantially different behavioral trends due to a unique medical condition, highlighting the need to consider individual differences when evaluating snake welfare. These results support our hypothesis and provide evidence that habitat modifications informed by ecology and natural history are successful in promoting speciestypical behaviors as indicators of positive welfare in snakes.
\end{abstract}

Keywords - Habitat design, Environmental exploration, Behavioral diversity, Reptile welfare, Environmental enrichment

A stimulating habitat that provides opportunities for a range of species-typical behaviors is crucial for promoting optimal welfare for animals living in the care of humans (Kagan et al., 2015; Melfie, 2009; Mellor et al., 2015). A significant change in zoo exhibit design over the past several decades has put an emphasis on constructing habitats based on the natural history of a species that accommodate natural behavioral repertoires and promote positive indicators of welfare, including increased behavioral diversity, environmental exploration, foraging and other species-typical behaviors (Mellor et al., 2015). After habitat modifications, it is important to conduct post-occupancy evaluations in 
order to determine how the changes impact animal behavior and other indicators of welfare (Kelling \& Gaalema, 2011).

Studies have shown that a stimulating environment that promotes species-typical behaviors improves reptile welfare as evidenced by positive behavioral indicators. Providing western chuckwallas (Sauromalus obesus obesus) with a rock feature that created additional choices for concealment led to decreased stereotypic behavior and increased use of retreats (Rose et al., 2014). Additionally, studies with both juvenile (Tetzlaff et al., 2018) and adult (Case et al., 2005) box turtles (Terrapene carolina) have shown that, given the choice, turtles preferred living in enriched, naturalistic environments, even when they were raised in simplistic and non-natural conditions. Habitat modifications including increased spatial complexity and the addition of climbing structures have also yielded positive behavioral results for corn snakes (Rose et al., 2014). However, research evaluating the impacts of modified/enriched environments for reptiles is still deficient, especially for snakes.

It is common folklore that snakes are sedentary animals that do not require spacious enclosures and, in fact, prefer confined spaces due to a fear of large, open spaces (Warwick et al., 2019). This apocryphal view of snake husbandry and welfare has often led to snakes being confined to small enclosures where they are unable to completely stretch out and assume a rectilinear body posture (Warwick et al., 2019; Wilkinson, 2015). Snakes exhibit a variety of different locomotion styles that are dependent on the snake's ability to fully extend the entire length of its body (Warwick et al., 2019). Being kept in an enclosure that does not allow full body extension inhibits a snake's ability to perform a full range of natural locomotion. As locomotion is an important part of the behavioral repertoire of any animal, providing the space needed to fully extend the body and properly locomote is important to ensuring optimal welfare for snakes. In addition, excessive inactivity can be a negative indicator of welfare for reptiles (Warwick et al., 2013). In highly exploratory snake species, increased locomotion and a modest increase in general activity levels can likely be interpreted as positive indicators of welfare, as it is important that normal behavior and activity are within "appropriate range and context" (Warwick et al., 2013). However, different species will vary in their use of space based on their ecology, and decisions about amounts and complexity of space need to reflect the natural history of the species.

Madagascar giant hognose snakes (Leioheterodon madagascariensis) are the largest of the hognose species, measuring up to $1.8 \mathrm{~m}$ when fully grown, and are endemic to the forests of Madagascar and its surrounding islands (Glaw \& Vences, 2007). Like all hognose snake species, they possess an upturned snout that is adaptive for digging through dense leaf litter while foraging for small amphibian prey (Degraaf \& Rudis, 1983; Glaw \& Vences, 2007). This morphological characteristic and the natural history of this species suggest that digging and other exploration behaviors are likely central to this species' welfare. Due to this species' active lifestyle and specific foraging style, increased locomotion as well as digging behaviors likely serve as indicators of positive welfare, so increased enclosure size and the addition of deep naturalistic substrate were included in the habitat modifications made as part of this study.

Behaviors of Madagascar giant hognose snakes were compared before and after habitat modifications to evaluate the effects of the changes on behavioral indicators of welfare, particularly increased locomotion, general activity and species-typical exploratory behaviors (digging and tongueflicking). The modified enclosures were larger in size and had cork bark furnishings and deep naturalistic substrate to provide opportunities for species-typical concealment, exploration and digging behaviors. We hypothesized that the snakes would show increases in behavioral diversity, overall activity levels and species-typical environmental exploration behaviors in the modified habitats indicating improved welfare.

\section{Methods}

\section{Subjects and Habitat Design}

The subjects of this study were five Madagascar giant hognose snakes from the same clutch living at the Holden Reptile Conservation Center at the Detroit Zoo in Royal Oak, MI, USA. The snakes 
were $2.9 \mathrm{yrs}$ old at the onset of data collection, approximately $0.9 \mathrm{~m}$ in length, and consisted of three males identified as 13256, 13257 and 13260, and two females identified as 13258 and 13259. Individual 13260 had a birth defect that made it necessary to remove one of his eyes shortly after hatching. Animal care staff gave 13260 daily medical treatments to manage his condition on an ongoing basis throughout the study. To facilitate healing, they also provided 13260 with an additional hide box filled with damp moss in both habitats, as well as occasional supplemental heat sources.

In both habitat conditions, all the snakes lived individually in nearly identical enclosures (Figure 1) in a behind-the-scenes area of the reptile center. The baseline habitats were stainless steel enclosures with opaque sides and glass lids. These habitats included newspaper for substrate and plastic hide boxes as furnishings. The modified habitats had glass fronts, opaque sides and more than twice the ground area of the original enclosures. The modified habitats included deep sand covered by a layer of mulch for substrate, along with cork bark as a furnishing, in addition to the plastic hide boxes, which were transferred with the snakes from their previous enclosures to provide a familiar element. Fluorescent UV bulbs on a 12:12 light dark cycle illuminated habitats in both conditions. The original habitats were climate controlled by ambient temperature set at $24.4^{\circ} \mathrm{C}$. A lamp provided supplemental heat to individual 13260. Modified habitats received supplemental heat from $53 \mathrm{~cm} \mathrm{x} 28 \mathrm{~cm}$ radiant heat panels set at $26.6^{\circ} \mathrm{C}$ above one side of each habitat. Care staff sanitized habitats on a regular schedule and provided consistent, identical diets to each individual throughout the study.
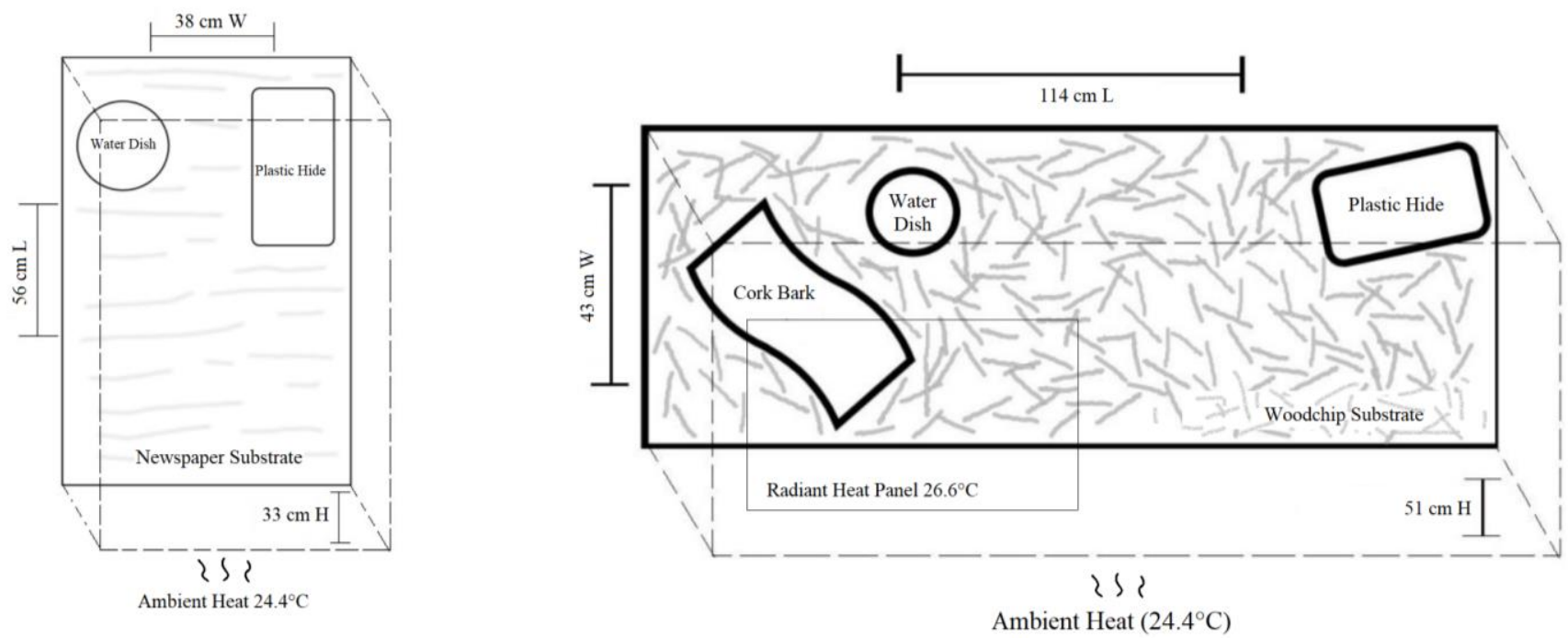

Figure 1. Top down views of the enclosures for Madagascar giant hognose snakes showing differences between the baseline enclosures (left) and the modified habitats (right).

\section{Behavioral Observations}

The first author conducted observations twice a day each Wednesday, Thursday and Friday within select time blocks in the morning (0800-1000 or 1000-1200 hrs) and afternoon (1200-1400 or 1400-1600 hrs) for eight weeks between March $29^{\text {th }}$ and May $26^{\text {th }}, 2017$. Morning and afternoon observation time blocks, as well as individual snake observation order within each time block, were randomized each day. All five individuals were observed consecutively in $10 \mathrm{~min}$ sessions (50 min total group observation) at variable start times within each randomized two-hour time block throughout the study. Data were not collected during the fifth week, which directly followed the transition between enclosures, in order to allow time for the snakes to acclimate to their new surroundings (Figure 2). The observer identified behaviors using an ethogram (Table 1) modified from behavioral studies on snakes (DeGraaf \& Rudis, 1983; Rose et al., 2014; Warwick et al., 2013). Randomized observation sessions utilized focal scan and all-occurrence sampling using ZooMonitor software (Ross et al., 2016; Wark et al., 
2019). The observer collected scan samples at 1-min intervals for behavior, exposure and furnishing use, as well as all occurrences of tongue-flicking and digging. Each individual was observed for a total of 460 $\min (7 \mathrm{hr}$ and $40 \mathrm{~min}): 230 \mathrm{~min}$ ( $3 \mathrm{hr}$ and $50 \mathrm{~min}$ ) of observation in each habitat.

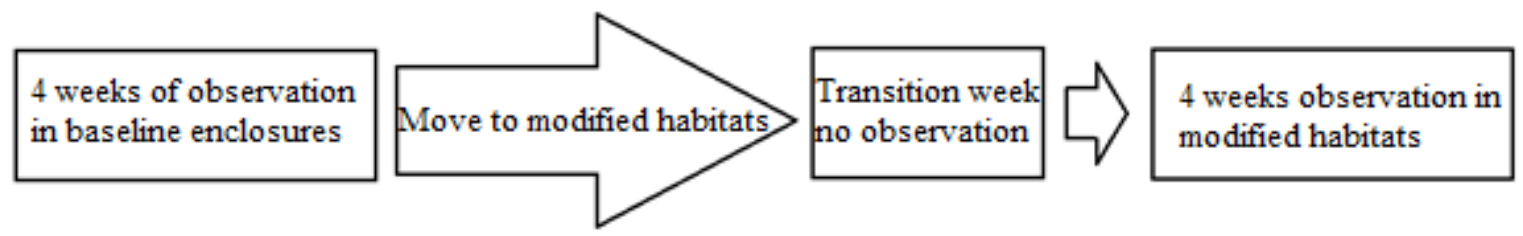

Figure 2. Timeline for behavioral observations.

Table 1

Ethogram of Madagascar Giant Hognose Snake Behaviors.

Activity Description

Behaviors

Locomotion

Digging*

Inactive

Investigation

Tongue-flicking*

Interacting with transparent boundaries

Other

Not visible

Exposure

Hiding

Exposed

Furnishing

Plastic hide box

Cork bark

None
When $>50 \%$ of body is undulating in any direction towards a new location

Animal is moving substrate aside with its snout

Animal is stationary and not displaying any notable behavior

Head and neck region move about in exploration of immediate surroundings (with or without tongue-flicking)

Chemosensory investigation in which the tongue moves swiftly in and out of the mouth

Head is actively in contact with a transparent boundary of the habitat

Animal is displaying a behavior not already described

Behavior being displayed by animal is not visible or is unclear

$>50 \%$ of body concealed from observer under substrate or furnishings

$>50 \%$ of body not concealed from observer under substrate or furnishings

Note. Modified from DeGraff \& Rudis, 1983; Rose et al., 2014; Warwick et al., 2013). All behaviors were used for scan sampling, and behaviors marked * were also monitored using all-occurrence sampling. 


\section{Data Analysis}

We calculated behavioral diversity from scan data using the Shannon-Weiner diversity index (Spellerberg \& Fedor, 2003). The formula for the index is:

$$
\mathrm{H}=-\sum\left(\mathrm{p}_{\mathrm{i}}^{*} \ln \left(\mathrm{p}_{\mathrm{i}}\right)\right)
$$

where $\mathrm{p}_{\mathrm{i}}$ is the proportion of time spent engaged in $i$ th behavior. Higher $\mathrm{H}$ values represent greater behavioral diversity resulting from either a greater variety of behaviors and/or a more even occurrence of behaviors.

We converted scan data to the percentage of time spent engaged in behaviors and calculated rates of tongue-flicking and digging per minute from the all-occurrence data. For analysis of scan data, we combined tongue-flicking, investigation and digging into a single behavior called 'exploration.' The behavior of 13260 often varied substantially from the other four snakes, perhaps due to his medical condition and husbandry routine. For this reason, we excluded 13260 from group analyses as an outlier, but he is still represented in individual data. All error bars represent the standard error for $N=4$ for group analyses (number of individuals) and $N=23$ for individual analyses (number of observation sessions).

We conducted statistical analyses using generalized linear mixed models (GLMM) in SPSS v. 25 (IBM Corporation, Armonk, NY). We created models for counts of scan and all-occurrence behaviors using Poisson distributions with log link functions, except for behavioral diversity and the logtransformed count of inactivity, which we analyzed using normal distributions with identity link functions. We created models with a random intercept and slope for individual ID*habitat condition to analyze behaviors, except in the following cases when a model would converge with a random intercept only: exploration, locomotion and inactivity. We created all models using a first-order autoregressive (AR1) covariance structure for the random effects. We adjusted degrees of freedom using a Sattherwhaite approximation and selected models based on the lowest Akaike Information Criterion (AIC) value. We report tests of fixed effects for condition for mixed models. We were unable to fit a mixed model for counts of all-occurrences of digging, so we analyzed this variable using a nonparametric Wilcoxon signed rank test. We also used Wilcoxon signed rank tests to examine individual trends in behavioral diversity, using the individual as the unit of analysis. For all tests, we consider results statistically significant when $p<.05$ and $.05 \leq p \leq .10$ to be statistical trends.

\section{Results}

\section{Behavioral Diversity}

As a group (excluding 13260), the snakes showed significantly greater behavioral diversity in the modified habitats compared to the baseline habitats (Table 2; Figure 3). Significant increases in average $\mathrm{H}$ value were observed between baseline and modified conditions for individuals 13258 (female; $N=23, Z$ $=-1.99, p=.04$ ), 13257 (male; $N=23, Z=-2.48, p=.01$ ), and 13256 (male; $N=23, Z=-2.23, p=.02$ ) (Figure 3). Although there was an increase in average $\mathrm{H}$ value for individual 13259 (female; $N=23, Z=$ $1.29, p=.19$ ), the difference was not significant. Individual 13260, the individual removed from group data, showed a decrease in $\mathrm{H}$ value (male; $N=23, Z=-0.56, p=.56$ ), though the decrease was not significant.

\section{Activity Budget (Scan Samples)}

Overall, the snakes showed a decrease in inactivity (increase in activity) and an increase in exploration and locomotion behaviors in the modified habitats. Time spent inactive decreased by $7.8 \%$ (i.e., time spent active increased) in the modified habitats (Table 2). Exploration (tongue-flicking, 
investigation and digging) increased by $6.3 \%$ and locomotion increased by $1.2 \%$ in the enriched habitats (Table 2).

\section{Exposure and Furnishing Use}

There was also a trend towards increased exposure in the modified habitats (Table 2). No differences in furnishing use were observed between habitats (Table 2).

\section{Table 2}

Summary Table Showing all Measures Taken (Behavioral and Space Use) During Observations, Average Percent Time Spent Engaged in Behaviors/space use Measure, and Significance Values Related to Differences Between Baseline and Modified Habitats (for the Group, Excluding 13260).

\begin{tabular}{|c|c|c|c|c|c|c|}
\hline $\begin{array}{c}\text { Sample } \\
\text { Type }\end{array}$ & Category & Sub-category & Pre-modification & Post-modification & Test Statistic & $\begin{array}{c}\text { Significance } \\
\text { Value }\end{array}$ \\
\hline \multirow{11}{*}{ Scan } & \multirow[t]{2}{*}{ Activity } & Inactive & $98.16 \% \pm 15.3 \%$ & $91.30 \% \pm 9.43 \%$ & $F(1,179)=17.61$ & $p<.001 * *$ \\
\hline & & Locomotion & $0.31 \%+0.38 \%$ & $1.58 \%+0.55 \%$ & $F(1,182)=5.86$ & $p=.02 * *$ \\
\hline & \multirow{3}{*}{ Exploration } & Tongue-flicking & $1.08 \% \pm 1.52 \%$ & $3.30 \% \pm 1.19 \%$ & \multirow{3}{*}{$F(1,179)=28.765$} & \multirow{3}{*}{$p<.001 * *$} \\
\hline & & Digging & - & $0.16 \% \pm 0.26 \%$ & & \\
\hline & & $\begin{array}{l}\text { Investigatory } \\
\text { Movements }\end{array}$ & $0.31 \% \pm 0.28 \%$ & $3.29 \%+1.03 \%$ & & \\
\hline & $\begin{array}{l}\text { Behavioral } \\
\text { Diversity }\end{array}$ & & $\mathrm{H}=0.43 \pm 0.08$ & $H=0.10 \pm 0.02$ & $F(1,21)=17.48$ & $p<.001^{* *}$ \\
\hline & \multirow{3}{*}{$\begin{array}{l}\text { Furnishing } \\
\text { Use }\end{array}$} & None & $20.52 \% \pm 11.63 \%$ & $15 \% \pm 3.59 \%$ & \multirow{3}{*}{$F(1,5)=1.83$} & \multirow{3}{*}{$p=.23$} \\
\hline & & Hide Box & $79.47 \% \pm 11.63 \%$ & $63.75 \% \pm 8.93 \%$ & & \\
\hline & & Cork Bark & - & $21.25 \%+7.04 \%$ & & \\
\hline & \multirow[t]{2}{*}{ Exposure } & Exposed & $13.54 \% \pm 11.27 \%$ & $29.56 \%+7.33 \%$ & \multirow[t]{2}{*}{$F(1,6)=4.955$} & \multirow[t]{2}{*}{$p=.07 *$} \\
\hline & & Concealed & $86.45 \%+11.27 \%$ & $70.43 \% \pm 7.33 \%$ & & \\
\hline \multirow[t]{2}{*}{$\begin{array}{c}\text { All } \\
\text { Occurrence }\end{array}$} & \multirow[t]{2}{*}{ Exploration } & Tongue-flicking & $\begin{array}{l}2.03 \pm 0.05 \text { tongue- } \\
\text { flicks } / \mathrm{min}\end{array}$ & \multirow{2}{*}{$\begin{array}{c}4.6 \pm 1.05 \\
\text { tongue-flicks } / \mathrm{min} \\
0.027 \pm 0.01 \\
\mathrm{dig} / \mathrm{min}\end{array}$} & $F(1,4)=34.02$ & $p=.003^{* *}$ \\
\hline & & Digging" $^{\#}$ & $0 \pm 0 \mathrm{dig} / \mathrm{min}$ & & $Z=-1.84$ & $p=0.07 *$ \\
\hline
\end{tabular}

Note. All tests are based on $n=4$ snakes. Behaviors were examined using generalized linear mixed models, except for \# which were analyzed using non-parametric Wilcoxon signed rank tests $* * p<.05$ and $* .05 \leq p \leq .10$.

\section{Tongue-flicking and Digging (All Occurrence Samples)}

The snakes showed a significant increase in rates of tongue-flicking (Table 2; Figure 4A), an important investigation behavior, in the modified habitats. Digging emerged as a common behavior after the move to the modified habitat, and differences in rates of digging between the two habitats were nearly significant using a Wilcoxon signed rank test (Table 2; Figure 4B) 


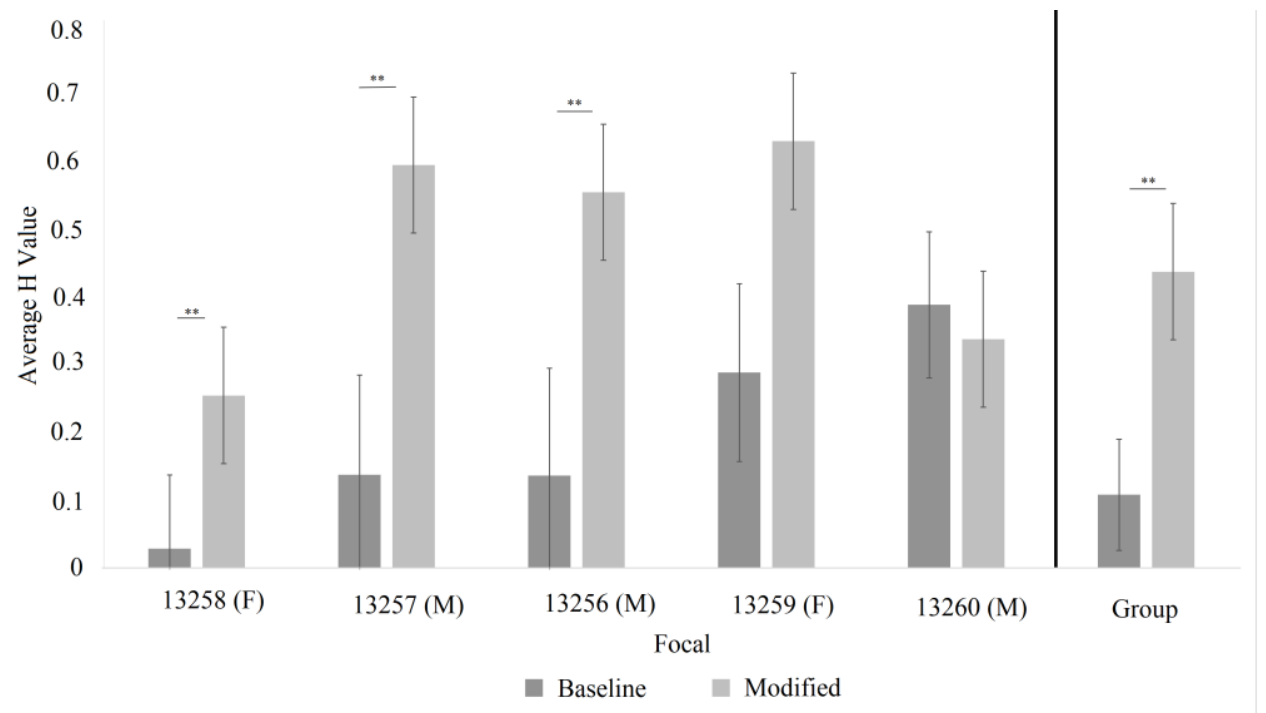

Figure 3. Average behavioral diversity $(\mathrm{H})$ values (mean $\pm \mathrm{SE}$ ) for Madagascar giant hognose snakes comparing baseline and modified habitats for each individual $(N=23)$ and the group $(N=4$, excluding 13260$)$. $* * p<.05, \mathrm{M}=$ male, $\mathrm{F}=$ female.
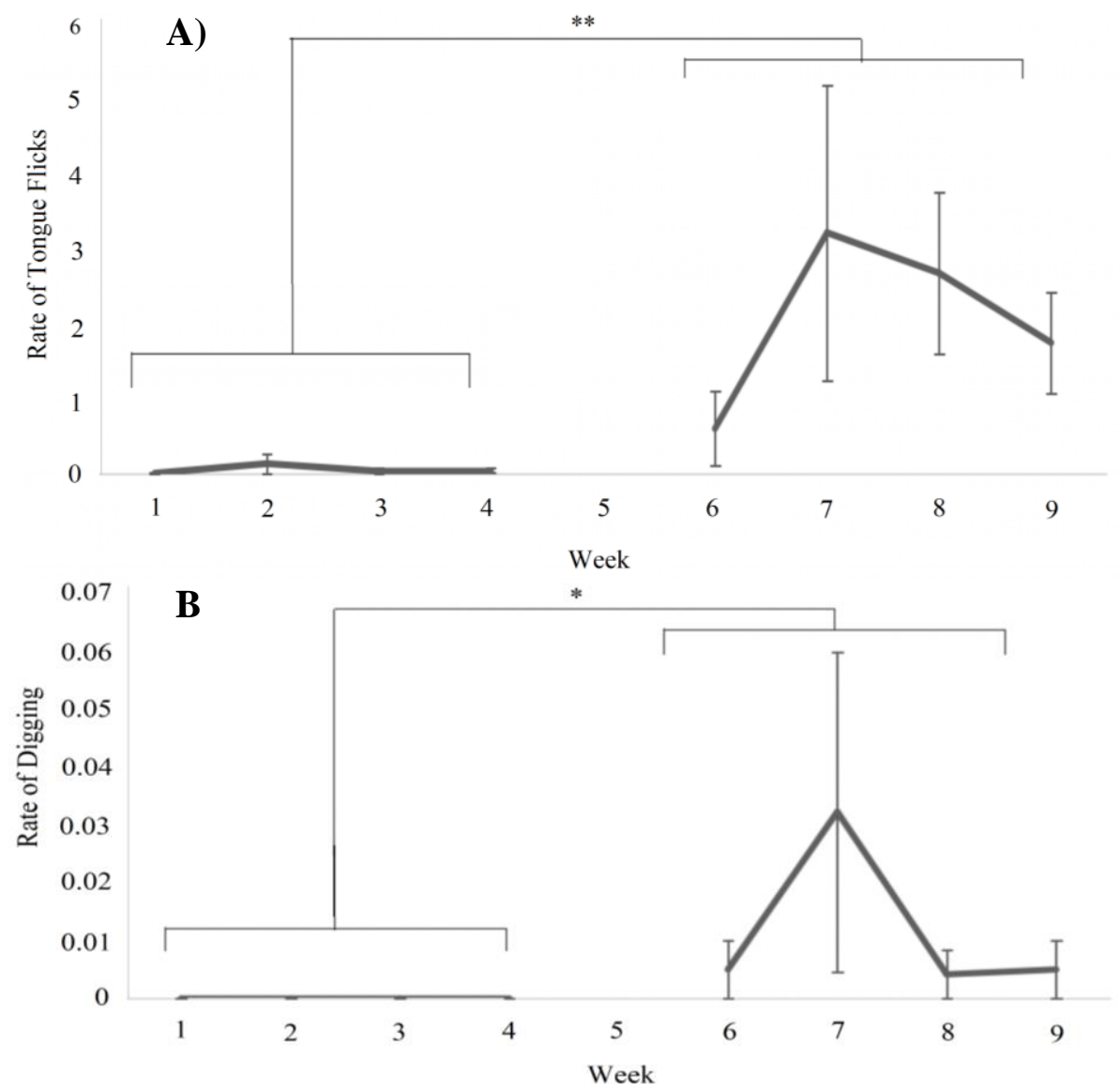

Figure 4. Average tongue-flicking (A) and digging (B) per minute (mean \pm SE) by week for $\mathrm{N}=4$ Madagascar giant hognose snakes. The snakes lived in the non-enriched habitats during weeks 1-4 and the enriched habitats in weeks 5-9. No data were collected immediately after their move, in week 5 , as indicated by the gap in each graph. $* * p<.05$ and $* .05 \leq p \leq .10$. 


\section{Discussion}

We observed behavioral changes suggesting that the habitat modifications positively affected the welfare of these Madagascar giant hognose snakes, as indicated by increases in behavioral diversity, overall activity, exposure time and exploration behaviors, including the emergence of digging behavior in the modified habitats. Behavioral diversity is becoming more common as a measure of welfare in a variety of species. Bashaw et al. (2016) evaluated behavioral diversity as a measure of welfare in a study of leopard geckos (Eublepharis macularius) and showed it increased in response to thermal, feeding and olfactory enrichment. The results of the present study are consistent with those of Bashaw et al. (2016), as the snakes exhibited greater behavioral diversity in the modified habitats, largely due to increases in exploration behaviors and locomotion. Miller et al. (2016) showed that behavioral diversity was negatively correlated with fecal glucocorticoid metabolite concentrations in cheetahs (Acinonyx jubatus), suggesting higher levels of behavioral diversity corresponded with reduced activity of the adrenal system. This physiological relationship supports the validity of behavioral diversity as an indicator of animal welfare. Additional non-behavioral measures such as a glucocorticoid analysis would have been helpful here to further confirm this result. Future studies of reptile welfare could attempt using non-invasive analysis of glucocorticoids or their metabolites in fecal samples or skin sheds (e.g., Berkvens et al., 2013) to incorporate another category of welfare indicators.

One source of increased behavioral diversity in the modified habitat was the emergence of digging behavior, which was not observed in the original enclosures (with the exception of a few bouts of digging in the newspaper by individual 13260, whose overall behavior patterns were different from the group). The appearance of digging behavior shows that the new substrate in the modified habitats provided a new opportunity to express this species-typical behavior and perhaps provided for a behavioral need. Behavioral needs have been defined as "positively motivated behaviors that are intrinsically pleasurable and promote biological functioning, such as foraging, exploration, grooming, and play" (Bracke \& Hopster, 2006, p .84). Benn et al. (2019) highlight how enrichment should target these highly motivated behaviors and that reptiles may respond much like carnivorous mammals, benefitting from increased behavioral opportunities. Digging is a behavior associated with foraging in hognose species, so it is likely to be a highly motivated behavior that is intrinsically rewarding to perform. The emergence of this behavior shows that the natural substrate in the modified condition provided an opportunity for the expression of a motivated behavior that may have been difficult with the newspaper substrate provided in the baseline enclosures.

Results indicated a trend for increased exposure in the modified habitats. Excessive hiding/concealment has been noted as a maladaptive behavior in captive reptiles that may indicate discomfort (Warwick et al., 2013), so increased exposure (while maintaining proper access to hides) may be a meaningful indicator of welfare in exploratory snake species. Rose et al. (2014) evaluated the effects of providing complex furnishings and natural plants on exposure time in corn snakes (Elaphe obsoleta), a highly exploratory species. Snakes in planted enclosures with complex furnishings that allowed for climbing and use of vertical space spent $31.7 \%$ of observed time exposed, while snakes in enclosures without complex furnishings or natural plants spent $98.6 \%$ of observed time concealed (1.4\% exposed). The snakes in the current study spent $13.54 \%$ of observed time exposed in the baseline habitats, compared to $29.56 \%$ in the modified habitats. These results support the conclusions of Rose et al. (2014), in that increased enclosure complexity leads to individuals spending less time concealed. In addition to complexity, the increased size of the modified enclosures may have given the snakes more options and space for activity, therefore increasing time spent exposed.

With the increase in observed time spent exposed, it is interesting that we did not see significant difference in furnishing use between conditions. Furnishings were described as 'in use' when a snake was under, on top of or in contact with either the plastic hide box or the new cork bark furnishings and described as 'exposed' when greater than $50 \%$ of their body was visible. This made it possible for both furniture use and exposure to be coded simultaneously and could account for the observed increase in exposure without a corresponding decrease in furnishing use. Providing animals with choice in their 
environment is essential to ensuring good welfare for captive animals (Kagan et al., 2015). Providing an additional choice of the cork bark furnishing, although it was not utilized as much as expected, could have contributed to increased rates of exposure and exploration. Alternatively, perhaps the cork bark was not a preferred furnishing, and the snakes would have used alternative furnishings more frequently if given the option. Benn et al. (2019) discuss the importance of preference tests for reptiles in which elements of the environment are evaluated for their effectiveness in promoting behavioral indicators of positive welfare. A preference test could be used to assess alternative furnishings for the snakes, providing information that could be used to inform future habitat modifications and design.

Appropriate enclosure size is particularly important for snake health and welfare. Whitehead (2018) cites confinement in restricted spaces as one of the leading causes of poor welfare experienced by snakes in captivity. Systematic studies on the effects of cage size are limited for reptiles, though numerous in other taxa. Warwick et al. (2019) evaluated 65 individual snakes and found that $37 \%$ assumed rectilinear body postures during a single hour of observation, suggesting that this behavior is common and that enclosures that allow for full extension of the body are crucial for snakes to be able to exhibit natural locomotion behaviors. We suggest that doubling the size of the enclosures in the current study, allowing for full body extension when it was not possible in baseline enclosures, may have been responsible for the observed increases in locomotion. These findings provide evidence that spacious enclosures that allow for a full range of locomotion may be critical to the welfare of snakes living in human care.

Tongue-flicking is an important chemosensory behavior in snakes and indicates that an animal is actively stimulated by and is exploring its environment. In this study, we observed a significant increase in rates of tongue-flicking between baseline and modified habitats, indicating that there was an increase in environmental exploration and engagement in the modified habitats, which could be an indicator of improved welfare. However, little is known about behavioral indicators of welfare in snakes, so, alternatively, increased tongue-flicking could also reflect stress associated with the move between enclosures. After stressful events such as handling or exposure to a novel environment, anolis lizards increase the use of their tongues to gather tactile and chemosensory information from the environment (Greenberg, 2002). It is possible that exposure to the modified habitats caused a similar increase in the snakes' arousal, resulting in the observed increase in tongue-flicking behaviors. However, the increase in tongue-flicking in this study was sustained over the post-modifications period, suggesting that the snakes were being consistently stimulated to explore their new environments, rather than experiencing a shortterm reaction to a novel environment and the move between enclosures. A longer-term observation period would be valuable in determining if the rate of tongue-flicking would be sustained over time in future studies, although it was not possible in this case.

It is also possible that differences in temperature between the baseline and modified habitats may have caused, in part, the observed increases in exploration behaviors and activity. Cooper and Vitt (1986) demonstrated that tongue-flicking rates in lizards are affected by temperature, with lowest rates of tongueflicking occurring between $15^{\circ} \mathrm{C}$ and $20^{\circ} \mathrm{C}$ and rates rapidly increasing to a peak at $30^{\circ} \mathrm{C}$. In a similar study, Brodie and Russell (1999) observed that juvenile garter snakes were quicker and more active at temperatures between $22.5^{\circ} \mathrm{C}$ and $30^{\circ} \mathrm{C}$ and slower and less active at temperatures between $15^{\circ} \mathrm{C}$ and $22.5^{\circ} \mathrm{C}$. Although there was only a small increase in temperature between the original habitats kept at $24.4^{\circ} \mathrm{C}$ and the modified habitats kept at $26.6^{\circ} \mathrm{C}$, it is possible that the increased activity levels observed in this study could have been caused, in part, by the temperature difference. Additionally, the supplemental heat source in the modified habitats was over only one side of the enclosure, potentially creating a heat gradient. This thermal gradient may have provided the snakes with an additional element of environmental choice. Reptiles are particularly dependent on the environment in order to maintain homeostasis, and much of their behavior can be explained by their motivation to regulate their body temperature. Perhaps the cause of the increase in locomotion was not simply the increased space but also the new opportunity to travel from one end of a thermal gradient to the other. Although it was not possible in this study, future studies should systematically control for temperature in order to rule it out as 
a factor shaping behavioral profiles and/or extend data collection to determine the significance of temperature gradients on captive reptile welfare.

In addition to differences in behavior in relation to changes in temperature, Brodie and Russell (1999) observed consistent individual differences that impacted the behavioral profiles of snakes. We saw a similar example in the behavior profile of individual 13260. This individual's behavior deviated from group trends in both habitats, and data collected on him was excluded from group analyses. Showing opposing trends to the other snakes, individual 13260 was less active, exhibited fewer exploration behaviors and had lower behavioral diversity in the modified habitat as compared to baseline. 13260 had an eye removed due to medical complications shortly after hatching, and it is possible that differences in medical condition, routine and housing could account for the differences in his behavior. Individual variation in temperament or his reduced sense of vision may also have resulted in the behavioral differences observed in this study (Brodie \& Russell, 1999). Results for this individual exemplify that welfare occurs at the individual level and that all habitat modifications and enrichment opportunities need to account for the specific needs of each individual.

There are several limitations to doing research in the zoo environment, to which this study is no exception. Like many studies, this project utilized only a single observer, and the nature of the study meant that she was aware of the hypotheses and likely had an expectation as to how the treatment would affect behavior. Although potential observer bias cannot be entirely ruled out in this case, operational definitions from the ethogram were consistently applied to avoid bias. Another limitation is that zoos generally house few individuals of many species, so opportunities for large sample sizes are limited, leading to reduced statistical power. For example, differences in rates of digging between habitats in this study only reached the level of a statistical trend, even though it was a new behavior that only occurred in the modified habitat. Therefore, the statistical significance likely did not reflect the true biological significance of this new behavior emerging when the opportunity arose. Finally, like many zoo studies, this study included several confounding factors, including an inability to distinguish between effects of enclosure size and complexity, as well as potential interference from differences in temperature regulation and gradients between the two habitats. We can determine the combined outcome of these factors given our data, but are unable to determine for certain which variable had an effect on specific behavioral responses. Confounding variables are a reality of conducting zoo research that should be minimized, but may not be fully removed. These realities should not preclude the goal of using the scientific process to generate evidence-based management practices for animals living in zoos.

\section{Conclusions}

1) The habitat modifications, especially increased enclosure size and the addition of deep sand/mulch substrate, were successful in promoting behavioral indicators of improved welfare for five Madagascar giant hognose snakes, as indicated by increases in behavioral diversity, species typical behaviors, overall activity levels and exploration behaviors.

a) The emergence of digging behavior indicates that the deep, natural substrate added during habitat modifications may have fulfilled a behavioral need unmet by the newspaper substrate in the original habitats.

b) Increased locomotion and overall activity levels suggest that the increase in enclosure size also had a positive impact on the snakes' welfare.

c) The observed increase in time spent exposed may be an indicator of improved welfare for this exploratory snake species. However, further information on how furnishing options affect exposure is needed to clarify this result.

2) Individual needs vary, and it is important to evaluate welfare on the level of the individual as well as the group. 
3) Habitat modification based on ecology and natural history is an effective method for promoting positive species-typical behaviors, health and welfare in snakes.

a) Even small changes to habitat design can have pronounced benefits for reptile welfare, meaning zoos can make meaningful differences in the lives of their resident reptiles, even if they are not in the position to make major habitat renovations.

\section{Acknowledgments}

This study would not have been possible without the cooperation of the wonderful and dedicated animal care staff at the Detroit Zoo. We would like to thank Tamara Colt for designing the habitats, for helping to conceptualize the study and for being a resource on snake husbandry. We would also like to thank Jeff Jundt for his assistance in conceptualizing the study.

\section{References}

Bashaw, M. J., Gibson, M. D., Schowe, D. M., \& Kucher, A. S. (2016). Does enrichment improve reptile welfare? Leopard geckos (Eublepharis macularis) respond to five types of environmental enrichment. Applied Animal Behavior Science, 184, 150-160.

Benn, A. L., McLelland, D. J., \& Whittaker, A. L. (2019). A review of welfare assessment methods in reptiles, and preliminary application of the Welfare Quality ${ }^{\circledR}$ protocol to the pygmy blue-tongue skink, Tiliqua adelaidensis, using animal-based measures. Animals, 9, 27.

Berkvens, C. N., Hyatt, C., Gilman, C., Pearl, D. L., Barker, I. K., \& Mastromonaco, G. F. (2013). Validation of a skin shed corticosterone enzyme immunoassay in the African house snake (Lamprophis fuliginosus) and its evaluation in the Eastern Massasauga rattlesnake (Sistrurus catenatus catenatus). General and Comparative Endocrinology, 194, 1-9.

Bracke, M. B. M. \& Hopster, H. (2006). Assessing the importance of natural behavior for animal welfare. Journal of Agriculture and Environmental Ethics, 19, 77-89.

Brodie, E. D., \& Russell, N. H. (1999). The consistency of individual differences in behaviour: Temperature effects on antipredator behaviour in garter snakes. Animal Behaviour, 57, 445-451.

Case, B. C., Lewbart, G. A., \& Doerr, P. D. (2005). The physiological and behavioural impacts of and preference for an enriched environment in the eastern box turtle (Terrapene carolina carolina). Applied Animal Behaviour Science, 92, 353-365.

Cooper, W. E. Jr., \& Vitt, L. J. (1986). Thermal dependence of tongue-flicking and comments on use of tongueflicking as an index of squamate behavior. Ethology, 71, 177-264.

DeGraaf, R. M., \& Rudis, D. D. (1983). Amphibians and reptiles of New England: Habitats and natural history. Amherst, MA: University of Massachusetts Press.

Glaw, F., \& Vences, M. (2007). A field guide to the amphibians and reptiles of Madagascar. Köln, Germany: Vences \& Glaw.

Greenberg, N. (2002). Ethological aspects of stress in a model lizard (Anolis carolinensis). Integrative and Comparative Biology, 42, 526-540.

Kagan, R.., Carter, S., \& Allard, S. (2015). A universal animal welfare framework for zoos. Journal of Applied Animal Welfare Science, 18, S1-S10.

Kelling, A. S., \& Gaalema, D. E. (2011). Postoccupancy evaluations in zoological settings. Zoo Biology, 30, 597610.

Miller, L. J., Pisacane, C. B., \& Vicino, G.A. (2016). Relationship between diversity and faecal glucocorticoid metabolites: A case study with cheetahs (Acinonyx jubatus). Animal Welfare, 25, 325-329.

Melfie, V. A. (2009). There are big gaps in our knowledge, and thus approach, to zoo animal welfare: A case for evidence-based zoo animal management. Zoo Biology, 28, 574-588.

Mellor, D. J., Hunt, S., \& Gusset, M. (Eds.). (2015). Caring for wildlife: The world zoo and aquarium animal welfare strategy. Gland, Switzerland: WAZA Executive Office.

Rose, P., Evans, C., Coffin, R., Miller, R., \& Nash, S. (2014). Using student-centered research to evidence-base exhibition of reptiles and amphibians: Three species-specific case studies. Journal of Zoo and Aquarium Research, 2, 25-32. 
Ross, M. R, Niemann, T., Wark, J. D., Heintz, M. R., Horrigan, A., Cronin, K. A., Shender, M.A., \& Gillespie, K. (2016). ZooMonitor (Version 3) [Mobile application software]. http://zoomonitor.org.

Spellerberg, I. F., \& Fedor, P. J. (2003). A tribute to Claude Shannon (1916-2001) and a plea for a more rigorous use of species richness, species diversity and the 'Shannon-Wiener' index. Global Ecology \& Biogeography, 12, 177-179.

Tetzlaff, S. J., Sperry, J. H., \& DeGregorio, B. A. (2018). Captive-reared juvenile box turtles innately prefer naturalistic habitat: Implications for translocation. Applied Animal Behaviour Science, 204, $128-133$.

Wark, J. D., Cronin, K. A., Niemann, T., Shender, M. A., Horrigan, A., Kao, A., \& Ross, M. R. (2019). Monitoring the behavior and habitat use of animals to enhance welfare using the ZooMonitor app. Animal Behavior and Cognition, 6, 158-167.

Warwick, C., Arena, P., Lindley, S., Jessop, M., \& Steedman, C. (2013). Assessing reptile welfare using behavioural criteria. In Practice, 35, 123-131.

Warwick, C., Arena, P., \& Steedman, C. (2019). Spatial considerations for captive snakes, Journal of Veterinary Behavior, 30, 37-48.

Whitehead, M. L. (2018). Factors contributing to poor welfare of pet reptiles. Testudo, 8, 47-61.

Wilkinson, S. L. (2015). Reptile wellness management. Veterinary Clinics: Exotic Animal Practice, 18, $281-304$. 